\title{
¿Qué factores se asocian con la reestrechez uretral en el paciente llevado a uretroplastia con o sin injerto?
}

\section{Which Factors are Associated with Urethral re-Stricture in Patient Submitted to Urethroplasty with or without Grafting?}

\author{
Ana María Ramos ${ }^{1}$ Diego Riatiga Ibáñez ${ }^{2}$ Andrea Bolaños ${ }^{3} \quad$ Luisana Castillo $^{4}$ Carlos Zapata 4 \\ Natalia Càrdenas Espinel ${ }^{5}$
}

${ }^{1}$ Residente de Urología, Fundación Universitaria de Ciencias de la Salud,

Address for correspondence Anamaria Ramos, Residente de Hospital de San Jose-Hospital infantil de San José, Bogotá, Colombia

2 Urólogo, Fundación Universitaria de Ciencias de la Salud, Hospital de San José- Hospital Infantil de San José, Bogotá, Colombia

3 Uróloga, Fundación Universitaria de Ciencias de la Salud, Hospital Infantil de San José, Bogotá, Colombia

${ }^{4}$ Residente de Urología, Fundación Universitaria de Ciencias de la Salud, Hospital de San José- Hospital Infantil de San José, Bogotá, Colombia

5 Interna, Fundación Universitaria de Ciencias de la Salud, Hospital de San José- Hospital Infantil de San José, Bogotá, Colombia

\begin{abstract}
Urología, Fundación Universitaria de Ciencias de la Salud, Hospital de San Jose-Hospital infantil de San Jose, Bogotá, Colombia (e-mail: anama319@hotmail.com; anamariaramos933@gmail.com).
\end{abstract}

Urol Colomb 2020;29:21-25.

\section{Resumen}

Palabras clave

- uretra

- uretroplastia

- estrechez uretral

- injerto
Objetivo Determinar los factores asociados a la reestrechez uretral en pacientes llevados a uretroplastia.

Métodos Estudio observacional analítico retrospectivo. Se revisaron las historias clínicas de pacientes llevados a uretroplastia en el HSJ entre 2012-2017. Se realizó un análisis bivariado para estudiar las asociaciones entre la reestrechez uretral posterior a la uretroplastia y las caracteristicas preoperatorias, intraoperatorias y posoperatorias, así como un modelo de regresión logística múltiple.

Resultados El total de pacientes llevados a uretroplastia fue de 82. La mediana de edad fue de 52 (RIQ = $35-67)$ años. De todos los pacientes incluidos en el estudio 29 $(36,2 \%)$ presentaron reestrechez. No se encontraron diferencias estadísticamente significativas en cuanto a los factores de riesgo en relación con reestrechez uretral en la población de estudio. Dentro de las técnicas quirúrgicas realizadas, los tipos de injerto utilizados en nuestros pacientes fueron la técnica con injerto de mucosa oral y con injerto de prepucio. De los pacientes a los cuales se les realizó uretroplastia con injerto de prepucio 13 (52\%), presentaron reestrechez mostrando una diferencia estadísticamente significativa $(p=0,02)$. El tiempo quirúrgico fue mayor en los pacientes con reestrechez uretral $(180 \mathrm{~min} \mathrm{RIQ}=120,0-189,0)$ comparado con los pacientes $\sin$ reestrechez $(140 \mathrm{~min} \mathrm{RIQ}=120,0-198,0)$. En cuanto al modelo de regresión logística, pacientes llevados a uretroplastia con injerto de prepucio poseen más riesgo de presentar reestrechez uretral comparado con los pacientes a los cuales se les realizó la uretroplastia con otro tipo de injerto o con una tecnica terminoterminal sin injerto OR $=2,91 \mathrm{IC} 95 \%(1,02-8,28)$. received

November 5, 2018

accepted

March 8, 2019
DOI https://doi.org/

$10.1055 / \mathrm{s}-0039-1685520$

ISSN 0120-789X.

e ISSN 2027-0119.
Copyright ( 2020 , Sociedad Colombiana License terms de Urología. Publicado por Thieme Revinter Publicações Ltda., Rio de Janeiro, Brazil. Todos los derechos reservados.

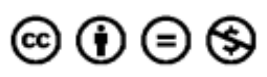


Conclusiones En nuestro estudio solo se encontró que el tipo de injerto utilizado es el único factor asociado al desarrollo de reestrechez uretral posterior a la realización de uretroplastia, otras variables clínicas descritas en la literatura no tuvieron asociación en nuestro estudio.

\section{Abstract}

\section{Keywords}

- urethra

- urethroplasty

- stricture

- graft
Objective To determine the association between risk factors, surgical procedure, and postoperative outcomes with stricture recurrence in patients who underwent urethroplasty.

Methods An analytical observational study with retrospective data collection was conducted. We reviewed the medical records of all the patients who underwent urethroplasty at HSJ between the years 2012-2017. The analysis of the data included a bivariate model to study the association between the risk factors, surgical procedure, postoperative outcomes and stricture recurrence. Then, we used logistic regression analysis.

Results 82 patients underwent urethroplasty: 29 (36.2\%) presented stricture recurrence. The median age was $52(\mathrm{IQR}=35-67)$ years old. There were no statistically significant differences between the risk factors, postoperative outcomes and stricture recurrence. In surgical technique we used buccal mucosal graft and penile skin graft. Stricture recurrence was observed in 13 patients who underwent penile skin graft urethroplasty showing a significant statistical difference $(p=0.02)$. The patients with stricture recurrence had longer operative time (180 minutes, IQR $=120.0-189.0$ ) compared to patients without ( 140 minutes, IQR $=120-198)$. The logistic regression analysis showed that patients who underwent penile skin graft urethroplasty had a greater risk of stricture recurrence OR $=2,91$ IC $95 \%(1,02-8,28)$.

Conclusions The type of graft used is the only factor associated with the development of stricture recurrence after performing urethroplasty. Other clinical variables described in the literature have no association with our study.

\section{Introducción}

La estrechez uretral afecta aproximadamente 1 de cada 10.000 hombres de 25 años y 1 de cada 1000 hombres con edad de 65 años o más ${ }^{1}$, la cual puede deberse a cualquier proceso que resulte de lesiones del tejido esponjoso subepitelial o del epitelio uretral, en las cuales se ha documentado la lesión iatrogénica o traumática como la etiología más común de estrechez uretral, la cual, al ser prolongada, puede llevar a complicaciones secundarias en el tracto urinario.

Se han documentado diferentes abordajes quirúrgicos a través del tiempo, y esencialmente se realizan en la actualidad estos dos principales: uretroplastia anastomótica para una estenosis corta, y uretroplastia de sustitución o de aumento, basadas en el uso de colgajos e injertos. ${ }^{2}$ A pesar de la literatura con la cual contamos a nivel mundial, existen pocos estudios de bajo poder sobre las comorbilidades y su influencia en los resultados de la uretroplastia ${ }^{3}$ así como las probabilidades de recurrencia relacionadas con el sitio, la longitud y la etiología de la re estrechez. ${ }^{4}$

\section{Métodos}

Estudio observacional analítico retrospectivo para el cual se revisaron las historias clínicas de pacientes llevados a uretroplastia en el HSJ entre enero del 2012 y diciembre del 2017. Para el análisis se excluyeron aquellos pacientes cuya historia clínica no permitía tener la información completa requerida por el protocolo de investigación así como menores de 18 años. Para el análisis estadístico, la información fue procesada con el paquete estadístico Stata 13 . Se reportaron frecuencias absolutas y porcentajes para variables cualitativas globales. Para variables cuantitativas, se aplicó la prueba de normalidad Shapiro Wilk para determinar la distribución normal o no normal, reportando las medidas de tendencia central y de dispersión correspondientes. Se realizó un análisis bivariado para estudiar asociaciones entre reestrechez uretral posterior a la uretroplastia y características preoperatorias, intraoperatorias, y complicaciones posoperatorias. Para este análisis, se definió como variable de desenlace, la reestrechez uretral en las diferentes categorías (SÍ/NO) reportando el valor p de la prueba Chi-cuadrado para variables cualitativas. Para las variables cuantitativas, se hizo un análisis de diferencias de medias o medianas entre grupos de acuerdo a la variable dependiente, reportando el valor p del estadístico de prueba correspondiente. Finalmente, se corrió un modelo de regresión logística forward para explicar la asociación encontrada en el análisis bivariado realizado previamente, utilizando una prueba de bondad de ajuste modelo (prueba de hosmerlemesshow), lo que muestra un ajuste estadístico de los datos. 


\section{Resultados}

El total de pacientes llevados a uretroplastia fue de 82 , de los cuales 29 (36,2\%) presentaron reestrechez. La mediana de edad al momento del estudio fue de 52 (RIQ = 35 -67) años (-Tabla 1). Dentro de los factores de riesgo descritos para reestrechez se encontraban tabaquismo $(31,7 \%)$, diabetes mellitus $(6,1 \%)$, y balanitis serótica obliterante $(1,2 \%)$, sin embargo, no se encontró una asociación con reestrechez uretral en la población de estudio ( - Tabla 1). En cuanto a la técnica quirúrgica realizada, los tipos de injerto utilizados fueron el de mucosa oral en $2(2,4 \%)$ pacientes e injerto de prepucio en 22 (28\%) pacientes de los cuales 13 (52\%) presentaron reestrechez mostrando una diferencia estadísticamente significativa $(p=0,02)$. En cuanto al tiempo quirúrgico, fue mayor en los pacientes con reestrechez uretral $(180 \mathrm{~min} \quad \mathrm{RIQ}=120,0-189,0)$ comparado con los pacientes sin reestrechez (140 min RIQ $=120,0-198,0)$ sin embargo, no se encontró una diferencia estadísticamente significativa. En cuanto al modelo de regresión logística, construido con variables significativas desde el punto de vista clínico y/o estadístico, se evidenció que los pacientes llevados a uretroplastia con injerto de prepucio, presentan más riesgo de presentar reestrechez uretral comparado con los pacientes a los cuales se les realizó la uretroplastia con otro tipo de injerto o con una técnica terminoterminal sin injerto OR $=2,91$ IC 95\% $(1,02-8,28)$ (-Tabla 2).

\section{Discusión}

La estrechez uretral en adultos es una patología común hoy en día. Las estadísticas reportadas en el Reino Unido y EE.UU, sugieren que los hombres se ven afectados con una incidencia creciente de 1 de cada 10000 hombres con edad de 25 años a diferencia de 1 por cada 1000 hombres con edad de 65 años o más. ${ }^{1}$ En Colombia, la incidencia exacta de su presentación es desconocida, pero sí queda claro que la morbilidad que la enfermedad agrega a esos pacientes es alta. ${ }^{5}$

El impacto económico de su manejo es significativo, y se estima un costo de aproximadamente 191 millones de dólares anuales, en los cuales el $70 \%$ de los gastos es explicado por diversos procedimientos diagnósticos y quirúrgicos requeridos para el manejo de esos pacientes. ${ }^{6}$ Se han descrito diferentes abordajes quirúrgicos que incluyen la uretrotomía interna endoscópica y esencialmente la uretroplastia anastomótica para una estenosis corta $\mathrm{y}$ uretroplastia de sustitución o de aumento basadas en el uso de colgajos e injertos. ${ }^{2}$ El injerto de mucosa oral fue descrito por primera vez para la reconstrucción uretral por Humby en 1941, mientras que Barbagli describió la uretroplastia de injerto dorsal en 1996 y desde entonces ella ha ganado importancia en el manejo quirúrgico de la estrechez. ${ }^{2-10}$

Se han mostrado tasas de éxito a largo plazo para la uretroplastia con injerto que oscilan entre el 73\% y el $92 \%$ $4,5,7-9$ y cabe resaltar que el tejido de mucosa oral se ha convertido en un sustituto uretral ideal debido a la facilidad
Tabla 1 Características preoperatorias, intraoperatorias y complicaciones

\begin{tabular}{|c|c|c|c|}
\hline \multirow{2}{*}{$\begin{array}{l}\text { Características } \\
\text { preoperatorias }\end{array}$} & \multicolumn{2}{|c|}{ Reestrechez $n$, (\%) } & \multirow[t]{2}{*}{$P$} \\
\hline & SI & NO & \\
\hline \multicolumn{4}{|l|}{ Factores de riesgo } \\
\hline Diabetes mellitus & $5(6,1)$ & $77(93,9)$ & 0,39 \\
\hline Tabaquismo & $26(31,7)$ & $56(68,2)$ & 0,86 \\
\hline $\begin{array}{l}\text { Balanitis serótica } \\
\text { obliterante }\end{array}$ & $1(1,2)$ & $81(98,7)$ & 0,43 \\
\hline \multicolumn{4}{|c|}{ Diagnóstico de estrechez uretral } \\
\hline Uretrocistografía & $18(35,2)$ & $33(64,1)$ & \multirow[t]{4}{*}{0,53} \\
\hline Cistoscopia & $5(41,6)$ & $7(58,3)$ & \\
\hline $\begin{array}{l}\text { Uretrocistografia+ } \\
\text { cistoscopia }\end{array}$ & $3(50,0)$ & $3(50,0)$ & \\
\hline Ecografía de pene & $1(100,0)$ & $0(0,0)$ & \\
\hline \multicolumn{4}{|c|}{ Localización de la estrechez } \\
\hline Uretra anterior & $18(32,7)$ & $37(67,2)$ & \multirow[t]{4}{*}{0,29} \\
\hline Uretra posterior & $5(62,5)$ & $3(37,5)$ & \\
\hline Bulbomembranosa & $6(46,1)$ & $7(53,8)$ & \\
\hline Prostatomembranosa & $0(0,0)$ & $1(100,0)$ & \\
\hline \multicolumn{4}{|l|}{ Causa de la estrechez } \\
\hline Paso de sonda & $7(58,3)$ & $5(41,6)$ & \multirow[t]{5}{*}{0,42} \\
\hline $\begin{array}{l}\text { Antecedente de cirugía } \\
\text { endoscópica }\end{array}$ & $6(54,5)$ & $5(45,4)$ & \\
\hline $\begin{array}{l}\text { Antecedente de } \\
\text { cirugía abierta }\end{array}$ & $2(25,0)$ & $6(75,0)$ & \\
\hline ETS & $1(20,0)$ & $4(80,0)$ & \\
\hline Trauma & $12(43,5)$ & $16(57,1)$ & \\
\hline \multicolumn{4}{|c|}{ Manejo previo a uretroplastia } \\
\hline Dilataciones & $2(28,5)$ & $5(71,4)$ & \multirow[t]{6}{*}{0,95} \\
\hline $\begin{array}{l}\text { Uretrotomía interna } \\
\text { endoscópica }\end{array}$ & $15(39,4)$ & $23(60,5)$ & \\
\hline Uretroplastia & $4(40,0)$ & $6(60,0)$ & \\
\hline Ninguno & $5(38,4)$ & $8(61,5)$ & \\
\hline $\begin{array}{l}\text { Dilataciones + } \\
\text { uretrotomía }\end{array}$ & $1(50,0)$ & $1(50,0)$ & \\
\hline Otros & $0(0,0)$ & $1(100,0)$ & \\
\hline \multicolumn{4}{|c|}{ Variables intraoperatorias } \\
\hline \multicolumn{4}{|l|}{ Tipo de injerto } \\
\hline Mucosa oral & $0(0,0)$ & $2(100,0)$ & \multirow[t]{3}{*}{0,02} \\
\hline Prepucio & $16(64,0)$ & $9(36,0)$ & \\
\hline Ninguno & $13(23,6)$ & $42(76,3)$ & \\
\hline \multicolumn{4}{|c|}{ Complicaciones posoperatorias } \\
\hline Infección & \multirow[t]{4}{*}{$8(80,0)$} & \multirow[t]{4}{*}{$2(20,0)$} & \multirow[t]{5}{*}{0,28} \\
\hline Hematoma & & & \\
\hline Dehiscencia & & & \\
\hline Fístula & & & \\
\hline Ninguna & $26(36,1)$ & $46(63,8)$ & \\
\hline
\end{tabular}




\begin{tabular}{|l|l|l|}
\hline Variable & \multicolumn{2}{|l|}{ Reestrechez uretral } \\
\hline $\begin{array}{l}\text { Características } \\
\text { preoperatorias }\end{array}$ & Mediana (RIQ) \\
\cline { 2 - 3 } & SI & NO \\
\hline Edad (años) & $53,0(33,0-67,0)$ & $52,0(38,0-65,0)$ \\
\hline IMC & $24,2(23,5-26,9)$ & $25,9(22,6-28,9)$ \\
\hline Características intraoperatorias \\
\hline $\begin{array}{l}\text { Tiempo } \\
\text { quirúrgico } \\
\text { (min) }\end{array}$ & $180,0(120,0-189,0)$ & $140,0(120,0-180,0)$ \\
\hline Sangrado (cc) & $200,0(100,0-400,0)$ & $100,0(100,0-300,0)$ \\
\hline Características posoperatorias \\
\hline $\begin{array}{l}\text { Tiempo uso } \\
\text { sonda (días) }\end{array}$ & $34,0(22,0-51,0)$ & $36,5(29,0-49,0)$ \\
\hline
\end{tabular}

Tabla 2 Regresión logística

\begin{tabular}{|l|l|l|l|}
\hline $\begin{array}{l}\text { Log likelihood }= \\
-48,291674\end{array}$ & \multicolumn{2}{l|}{ Number of obs $=76$} \\
\hline Prob $>$ chi2 $=0,2149$ & \multicolumn{2}{l|}{ Pseudo $R 2=0,0442$} \\
\hline Reestrechez & OR & P & IC 95\% \\
\hline Tipo injerto & 2,91 & 0,04 & $1,02-8,28$ \\
\hline Prepucio & 2,42 & 0,39 & $0,31-18,90$ \\
\hline Mucosa oral
\end{tabular}

de obtención, características del procedimiento quirúrgico, ausencia de folículos pilosos, compatibilidad en un ambiente húmedo así como el crecimiento y supervivencia del injerto. Debido a esas características únicas, la mucosa oral ha ganado importancia en la urología reconstructiva.

Por otra parte, la uretroplastia con injerto de prepucio descrita en 1953 por Presman y Greenfield, y posteriormente popularizada por Devine, ha ido ganando importancia paulatinamente con resultados comparables al injerto de mucosa oral, sin embargo, la escogencia del tipo de injerto ya sea de mucosa oral o prepucio depende de indicaciones específicas. ${ }^{11}$ En adultos, la uretroplastia sigue siendo el gold estándar en el manejo de la estrechez uretral, y las tasas de reestrechez a largo plazo varían del $1,2 \%$ al $14 \%$ para la uretroplastia anastomótica y del $58 \%$ para la uretroplastia de sustitución ${ }^{12}$ con una tasa de recurrencia de hasta el $21 \%{ }^{13}$

Al comparar nuestros resultados con estudios previos, en cuanto al tipo de injerto utilizado durante la uretroplastia, se encontró una diferencia estadísticamente significativa OR 2.91 IC $95 \%(1,02-8,28)$, en la cual los pacientes llevados a uretroplastia con injerto de prepucio presentaron más riesgo de reestrechez uretral, comparado con pacientes a los cuales se les realizó uretroplastia con mucosa oral o la técnica terminal sin injerto. Se puede observar que la uretroplastia con injerto de prepucio a largo plazo, tiene una menor tasa de éxito comparado con la uretroplastia con injerto de mucosa oral o la no utilización del mismo; aunque hay estudios contradictorios en cuanto a esos resultados. ${ }^{14-16}$

Este trabajo tiene el objetivo de determinar los diferentes factores asociados a la reestrechez uretral en pacientes llevados a uretroplastia con o sin injerto, entre los cuales se encuentran la diabetes mellitus, tabaquismo, y la balanitis serótica, así como el tipo de injerto, la longitud, y etiología de la estechez ${ }^{17-19} \sin$ embargo, no se encontraron diferencias estadísticamente significativas en nuestra casuística, así como tampoco se encontró asociación con factores como el tiempo de sondaje vesical, calibre de la sonda utilizado posterior al procedimiento, ni el manejo previo a la realización de la uretroplastia.

Es importante mencionar las limitaciones del estudio. Es un estudio retrospectivo el cual fue realizado en una sola institución, con una casuística limitada, por lo cual los resultados no son extrapolables a otras poblaciones; otra de las limitaciones, fue la pérdida de datos por la cual no se pudieron incluir en su totalidad variables a analizar de importancia de nuestro estudio. A pesar de esas limitaciones, y dado a que en Colombia no se han publicado estudios en los cuales se muestren esas asociaciones, este estudio representa un punto de partida para futuras investigaciones en el tema.

\section{Conclusiones}

Nuestro estudio sugiere que el tipo de injerto utilizado es un factor asociado al desarrollo de la reestrechez uretral posterior a la realización de la uretroplastia. Otras variables clínicas descritas en la literatura (factores de riesgo, intraoperatorias y posoperatorias), no tuvieron asociación con el riesgo de reestrechez.

Conflicto de Intereses

Los autores declaran no tener ningún conflicto de intereses.

\section{Agradecimientos}

Los autores agradecen a Juan Pablo Alzate por su invaluable asistencia en el análisis estadístico del estudio.

\section{Referencias}

1 Mundy AR. Management of urethral strictures. Postgrad Med J 2006;82(970):489-493

2 Spilotros M, Sihra N, Malde S, et al. Buccal mucosal graft urethroplasty in men-risk factors for recurrence and complications: a third referral centre experience in anterior urethroplasty using buccal mucosal graft. Transl Androl Urol 2017;6(03):510-516

3 Breyer BN, McAninch JW, Whitson JM, et al. Multivariate analysis of risk factors for long-term urethroplasty outcome. J Urol 2010; 183(02):613-617

4 Lozano Ortega JL, Pertusa Peña C. [Surgical treatment of urethral stenosis. Results of 100 urethroplasties]. Arch Esp Urol 2009;62 (02):109-114

5 Andrich DE, Mundy AR. What is the best technique for urethroplasty? Eur Urol 2008;54(05):1031-1041

6 Lopera Toro AR, Hessen M, Gaviria J, et al. Manejo de la estenosis de uretra anterior con técnica combinada: reporte de cuatro casos. Urol Colomb 2013;22(02):43-48

7 Micheli E, Ranieri A, Peracchia G, Lembo A. End-to-end urethroplasty: long-term results. BJU Int 2002;90(01):68-71

8 Barbagli G, De Angelis M, Romano G, Lazzeri M. Long-term followup of bulbar end-to-end anastomosis: a retrospective 
analysis of 153 patients in a single center experience. J Urol 2007; 178(06):2470-2473

9 Santucci RA, Mario LA, McAninch JW. Anastomotic urethroplasty for bulbar urethral stricture: analysis of 168 patients. J Urol 2002; 167(04):1715-1719

10 Zimmerman WB, Santucci RA. Buccal mucosa urethroplasty for adult urethral strictures. Indian J Urol 2011;27(03):364-370. PMC. Web. 30 Nov. 2017

11 Raber M, Naspro R, Scapaticci E, et al. Dorsal onlay graft urethroplasty using penile skin or buccal mucosa for repair of bulbar urethral stricture: results of a prospective single center study. Eur Urol 2005;48(06):1013-1017

12 Han JS, Liu J, Hofer MD, et al. Risk of urethral stricture recurrence increases over time after urethroplasty. Int J Urol 2015;22(07): 695-699

13 Kinnaird AS, Levine MA, Ambati D, Zorn JD, Rourke KF. Stricture length and etiology as preoperative independent predictors of recurrence after urethroplasty: A multivariate analysis of 604 urethroplasties. Can Urol Assoc J 2014;8(5-6):E296-E300
14 Andrich DE, Dunglison N, Greenwell TJ, Mundy AR. The long-term results of urethroplasty. J Urol 2003;170(01):90-92 [Internet]

15 Marchal C, Pérez JE, Herrera B, et al. Barbagli's dorsal urethroplasty. Analysis of results and factors for success. Arch Esp Urol 2010;63(07):537-544

16 Fichtner J, Filipas D, Fisch M, Hohenfellner R, Thüroff JW. Longterm outcome of ventral buccal mucosa onlay graft urethroplasty for urethral stricture repair. Urology 2004;64 (04):648-650

17 Redón Gálvez L, Escudero RM, Álvarez Ardura M, Otaola Arca H, Alarcón Parra RO, Páez Borda A. Factores predictivos de recidiva de estenosis uretral tras uretrotomía endoscópica. Actas Urol Esp 2016;40(08):529-533

18 Barbagli G, Kulkarni SB, Fossati N, et al. Long-term followup and deterioration rate of anterior substitution urethroplasty. J Urol 2014;192(03):808-813

19 Belsante MJ, Zhao LC, Hudak SJ, Lotan Y, Morey AF. Cost-effectiveness of risk stratified followup after urethral reconstruction: a decision analysis. J Urol 2013;190(04):1292-1297 